\title{
Surgical treatment in obstructive sleep apnea syndrome: an overview
}

\author{
Lucrezia Sparacino $^{1 *}$, Luca Bruschini ${ }^{1}$, Andrea De Vito ${ }^{1}$, \\ Giacomo Fiacchini ${ }^{1}$, Stefano Berrettini ${ }^{1,2}$
}

${ }^{1}$ ENT, Audiology and Phoniatrics Unit, University Hospital, Pisa, Italy

${ }^{2}$ Division of ENT Diseases, Karolinska Institutet, Stockholm, Sweden

Received: 10 May 2020

Revised: 01 July 2020

Accepted: 04 July 2020

\section{*Correspondence:}

Dr. Lucrezia Sparacino,

E-mail: lucreziasparacino@gmail.com

Copyright: (c) the author(s), publisher and licensee Medip Academy. This is an open-access article distributed under the terms of the Creative Commons Attribution Non-Commercial License, which permits unrestricted non-commercial use, distribution, and reproduction in any medium, provided the original work is properly cited.

\begin{abstract}
Obstructive sleep apnea syndrome is a common pathology affecting pediatric and adult population. It is caused by an increase upper airway resistance due to augmented collapsibility that cause alteration in ventilation and oxygen saturation. Sleep oxygen desaturations have multi-organ implications. Since many years, OSAS, has been recognized as a risk factors for comorbid cardiovascular disease. Well-known is the causal link with cognitive impairment and atherosclerosis. Healthcare professionals, should be trained to recognize signs and symptoms of the disease in order to send the patients forward for specialist care in centers dealing with sleep-disordered breathing. However treatment knowledge is of pivotal importance in the cultural baggage of all the physicians. Surgical and non-surgical therapies have undergone a great number of changes. Going along with the innovations in diagnostic evaluation and phenotyping of the patients, surgeries were heightened by new developments such as advances in tailoring procedures and technologies. The aim of this overview is to describe the evolution and main characteristics of encoded procedures classified into site of surgical correction.
\end{abstract}

Keywords: Obstructive sleep apnea syndrome, OSAS, Surgery

\section{INTRODUCTION}

Obstructive sleep apnea (OSAS) is a pathology of the upper airways causing the interruption of airflow during sleep time. The presence of apneas or hypopneas can cause a reduced blood oxygenation that can lead to a hard overload of the cardiovascular system. In chronic this would produce a right hearth failure (mostly with preserved left ejection rate) and damage the delicate structure of the lungs vessels and parenchyma. Not only limited to the cardiovascular system OSAS is associated with central nervous system degenerative lesions and dementia. ${ }^{1}$ The clinical manifestations are multiple and various: someone can experiment a huge sleepiness, others can even notice their condition. Suspect can be set by all the physicians but diagnosis is carry out by an OSAS expert through clinical history, objective examination and a sleep monitoring test. Overnight testing (polisomnography) is a sleep recording in which a control unit check 13 channels including EEG, cardiac frequency and $\mathrm{O}_{2}$ blood saturation, thorax and abdominal movements, nasal airflow and position. During all sleep time a video control is managed by a technician that write down a report. The complete polysomnography need a hospitalization that in most cases isn't achievable for high costs. Therefore alternative methods have been developed, using less channels, allowing the patients to sleep at home. The results are expressed by Apnea hypopnea index (AHI) that is the summation of all the apnoic and hypopnoic events for hours of sleep. OSAS is diagnosed if AHI $>5$ episodes. The severity was defined mild if $5<\mathrm{AHI}<15$, moderate if $15<\mathrm{AHI}<30$ and severe if AHI $>30$ according to the AASM 2017 guidelines. ${ }^{2}$ All the patients with comorbidities (cardiovascular and cerebrovascular disease, type 2 diabetes, metabolic 
syndrome, asthma, COPD, cancer) need a careful management and the more effective treatment as possible in order to reduce OSAS negative prognostic impact. ${ }^{3}$ Treatment options vary according to the characteristics of the patient and attempt to widen the upper airways in order to reduce collapsibility: continuous positive airway pressure (CPAP), postural therapy, oral appliances and surgery. Continuous positive airway pressure (CPAP) apply a positive pressure to the upper airways causing a dilatation of all the collapsible structures and it is considered the gold standard treatment. The pressure is conveyed through a mask that is applied on the nose and mouth (or only on the nose). The objective results are closely linked to the compliance of the patient. ${ }^{4}$ Other therapies like postural therapy or oral appliances offer a possibility of treating some very selected OSA patient suffering from postural apneas and from a collapse that is solvable by moving forward the mandible. ${ }^{5}$

Surgical treatment, instead, is not influenced by the patient's compliance and, if the procedure is lead on by a skilled surgeon, and all the perioperative precautions are implemented, is safe and burden by little complications. Tracheotomy was the first surgical procedure proposed for OSAS patients in the perspective of bypassing a severe obstruction (Hall 1848, Kuhl 1968). ${ }^{6-7}$ Until that moment the anatomical and clinical studies provided a solid base to create tailored surgical answers based on the site of obstruction. Indeed the most challenging diagnostic step is to identify site and characteristics of the collapsible airways; for this, objective examination with endoscopy and specific maneuvers (Müller's manoeuvre, mandibular active and passive advancement) could give the physician information about all the collapsible sites. If the site of obstruction is not clearly recognizable, drug induced sleep endoscopy (DISE) can be proposed. DISE (first described by Croft and Pringle in 1991) is an operating room procedure that can evidence the different obstructive sites during a sleep that is close as possible to the physiological sleep. ${ }^{8}$ Identifying the site of obstruction can allow the physician to design a tailored surgery.

The aim of this article was to synthetically analyze the most common OSAS surgical procedure providing a brief description and indications, knowledge that have to be part of every surgeon cultural heritage.

\section{NASAL SURGERY}

Some of the first surgical procedures proposed during centuries for the treatment of OSAS is nasal surgery. Hippocrates firstly described a procedure of nasal polyps extraction to reduce snoring. In the $19^{\text {th }}$ century many scientist concentrated their attention on the importance of adenoids and tonsils (Meyer, Betz) in the pathogenesis of snoring and apneas., ${ }^{9,10}$ The heritage of nasal isolated responsibility for snoring and apneas unfortunately pervades nowadays management. Nasal surgery alone is not a proper therapy for OSAS for the simple concept that the nose isn't a collapsible segment. Proposing nasal procedures to a patient have to be included in a perspective of a multistep approach. For example, the correction of nasal dysmorphia (septoplasty with or without turbinoplasty) should be considered a part of therapy, for instance in a CPAP treated patient to facilitate the application of a nasal mask instead of the oronasal mask. $^{11,12}$

\section{OROPHARYNGEAL SURGERY}

Protagonist through the ages the oropharynx is the most frequently involved site of collapse of the upper airways. ${ }^{3}$ Oropharyngeal collapse can be evaluated by fibroendoscopic view during the Müller maneuver and can be classified into anteroposterior, lateral or transverse and circular closure with a scale of four degrees. ${ }^{13}$ These characterization is fundamental for the correct surgery procedure that can produce a stiffened palate and can move forward (through specific vectors, anteriorly or laterally) the palate.

The first procedure described was the "Palatoplasty and uvulectomy" performed in 1963 by Ikematsu Takenosuke. ${ }^{14}$ The subsequent studies of Quesada, that verify with polysomnographic objectivation, the results of the modified surgical procedure by Ikematsu inroads in sleep obstructive syndrome the attention on oropharynx and, due to later anatomical insights, permits the elaboration of multiple techniques. ${ }^{15}$ The most performed surgical procedure called 'uvulo-palato-pharyngoplasty (UPPP or $\mathrm{UP}_{3}$ )' was introduced by Shiro Fujita in 1981 and remained for many years a point of reference, but the wound healing lead not infrequently to nasal reflux, difficult swallowing and a sensation of oropharyngeal foreign body. ${ }^{16}$ The procedure consist in a three step surgery: the first step is a tonsillectomy; then the mucosal and submucosal tissue of the glossopalatal arch is removed toward the tonsillar fossa, while the underlying muscular layer is preserved. The third step is the trimming of the palatopharyngeal mucosa that is elevated and sutured anteriorly to the corresponding strip with adsorbable wire. Less invasive is 'anterior palatopharyngoplasty' introduced by Ellis in 1994 and modified by Pang et al. ${ }^{17,18}$ It consist in a horizontal rectangular strip of mucosa removal from the soft palate variable in length and width. The muscular visible surface is then covered by suturing the mucosal flaps with an adsorbable wire.

For transverse closure patterns was proposed the 'lateral expansion pharyngoplasty' first described by Cahali then modified by Pang and Woodson in 2007. The technique consists in a bilateral tonsillectomy and a palatopharyngeal muscle antero-supero-lateral-rotation flap sutured through the muscle bulk. ${ }^{19,20}$ In 2016 Pang et al suggest the association of anterior pharyngoplasty and lateral expansion pharyngoplasty in patients with concentric collapse. ${ }^{21}$ 
The most important innovation in palatal technique was the introduction of barbed suture conveyed from orthopedic, urologic surgery and used in facial lifts. This type of thread has barbs along that allows the surgeon to fix the tissue without knots. Using this new wires anterior palatoplasty was afterwards modified by Mantovani in 2012 that introduced a suspension surgery called 'Roman blinds' or VULP (velo-uvulo-pharyngeal lift): the mucosa and connective tissue of the soft palate is anchored to the posterior nasal spine and to the hamulus bilaterally and stiffen by a multiple trans mucosal along a semicircular way from the uvula to the posterior tonsillar pillar. ${ }^{22-23}$

Vicini et al modified the Roman blinds procedure introducing 'Barbed repositioning pharyngoplasty' (BRP) and extend surgical indication to moderate and severe OSAS patients with concentric closure pattern. The procedure begin with a bilateral tonsillectomy then a barbed thread is introduced in the centre of the soft palate (corresponding to the infero-posterior nasal spina) and passed laterally around the pterygomandibular raphe. The needle enter close to the exit point and enter the tonsil bed, then through the palatopharyngeal muscle (previously transected in its inferior part) and comes out in the mucosa of the posterior pillar. The posterior pillar is entered, the needle pass through the tonsil bed,3 and comes out at the raphe. Uvular tip can be partially resected. $^{24}$

Latest proposal dates back to 2017 when introduced the 'alianza' surgical method born from a combination of anterior and lateral pharyngoplasty with barbed suture. ${ }^{25}$ The patients were all tonsillectomized: a rectangular area of mucosa is striped to expose the muscular plan of the soft palate as in Pang's procedure; postero-inferior nasal spina and hamulus were the anchorage structures and a suture of the palate, like in BRP, is done without transecting and isolating the palatopharyngeal muscle.

\section{HYPOPHARYNGEAL SURGERY}

Hypopharynx is the second most frequently involved site of obstruction. ${ }^{3}$ The first proposal of a multilevel oropharyngeal and hypopharyngeal surgery was made by Djupesland with the 'Palatopharyngealglossoplasty' in 1987 focusing also on the tongue base and hypopharynx a possible appointed site of surgery. ${ }^{26}$ In 1994 the Stanford group encode the hyoid suspension that purpose is to moving the hyoid bone antero-inferiorly anchoring to the thyroid cartilage through a suture: once performed the skin incision, dissection is done until the plane of the thyroid cartilage and the surface of the hyoid bone were exposed and the thyrohyoid membrane was clearly defined. A non adsorbable suture thread is wrapped around the body of the hyoid bone on each side of the midline with a sharp needle and then pierce $1 \mathrm{~cm}$ trough the upper border of the cartilage. The sutures were tied steadily and gently, with the neck in neutral position in order to approach hyoid to thyroid cartilage and stiff the collapsible segment. ${ }^{27}$ In 1999 Chabolle proposed an open tongue tonsil resection but the high risk of preoperative complication associated with the still poor phenotyping lead to little use of this technique allow some physicians to prefer less invasive, proposed by less effective, techniques (f.i. radio frequency Powell 1999) ${ }^{28,29}$ In 2010 Vicini et al first suggest the feasibility of a tongue reduction in moderate to severe OSAS patients by 'trans oral robotically assisted surgery'. ${ }^{30}$ Surgical indications included airway obstruction caused by an antero-posterior closure pattern due to tonsillar tongue base hypertrophy in $>18$ years moderate to severe OSAS patients. Increased morbidity, procedural costs and operating room time affects in a negative way the robotic technique that has nevertheless utterly improved the vision of the surgical field and the surgeon's movements. As described by Vicini et al once the DaVinci is settled, the surgeon explore the field from epiglottis and tongue base. Then a piecemeal resection begin from the medial to the lateral margin of the lingual tonsil farther from papillae circumvallate and the upper third of the suprahyoid epiglottis (above the pharyngoepiglottis folds, to minimize the risk of aspiration) can be removed giving attention to the preservation of the recurrent laryngeal branches. Controversial is the possibility to perform a tracheostomy in order to protect the airways during the immediate postoperative period especially if tongue procedure is ancillary to tonsillectomy or epiglottectomy. As reported before TORS is a multistep surgery that could be associated to other oropharyngeal approaches (BRP) or bimaxillary advancement depending on the sites of obstruction. ${ }^{31}$

\section{LARYNGEAL SURGERY}

The cartilaginous structure of larynx keep safe from the collapse. The only segment that could be rarely involved in OSAS is epiglottis. In this phenotype every breath when realized a negative pressure in the upper airways, the epiglottis overturns on the aditus locking inspiration. This mechanism is due to the laxity of the suspensory structures. This phenotype is one of the rarest but is important of recognize this condition, also during clinical examination, because is an absolute contraindication to the CPAP (the positive pressure should push the epiglottis above the aditus). ${ }^{3,32}$ Epiglottopexy was first described by Fearon and Ellis in 1971 the surgery was oriented to correct laryngomalacia in a pediatric patient. ${ }^{33}$ Epiglottoplasty was indeed described by Lane et al in 1984. This surgery reshapes laryngeal structure by trimming away excessive tissue from the arytenoid cartilages, arytenoid folds, and lateral edges of the epiglottis. ${ }^{34}$ With improving robotic technologies this surgery could be done transorally with robotic assistance in some cases as a part of a multilevel approach. In 2019 Salamanca et al encoded a new non resective procedure called 'epiglottis stiffen operation'. After exposition of the epiglottis cauterization of the lower half of the lingual side is done between the glosso-epiglottic folds. Healing process help to create a retractive tissue that approximate epiglottis to tongue base and stiffen the hypopharynx. ${ }^{35}$ 


\section{TRACHEOSTOMY}

The first treatment for OSA syndrome reported in literature was tracheostomy (Hall 1848, Kuhl 1968). ${ }^{6,7}$ The progression in phenotyping and the surgical success allow the physicians to avoid this aggressive surgical method. Nevertheless it can be used in selected cases that need urgent treatment in order to plan a multilevel approach.

A $2 \mathrm{~cm}$ horizontal incision is made above the jugular notch, then a plan dissection is done to identify the prelaryngeal muscles (sternohyoid and sternothyroid muscles) that are retracted laterally to identify the trachea. Depending on the position of the thyroid gland and the abilities of the surgeon a suprathyroid, intrathyroid or infrathyroid tracheostomy is performed. A Bjork flap or an $\mathrm{H}$ shaped incision is performed in the anterior wall of the trachea and a fenestrated tube is positioned. However literature reports reduced symptoms and successful reduction in AHI, tracheostomy submit the patient to aesthetic and medical difficulties (like an augmented risk of pulmonary infections) that should be limited by other surgical procedures. ${ }^{36}$

\section{HYPOGLOSSAL STIMULATION}

Hypoglossal stimulation (HS) can be used for moderate to severe OSA patients that express an intolerance to CPAP and a morphological pattern of anteroposterior collapse. ${ }^{37}$ The objective is that hypoglossal fibers' stimuli are responsible of the contraction of genioglossus muscle that cause the protrusion and stiffening of tongue. Many companies have developed devices relying on this concept with some kind of differences among different tools. The one proposed by Inspire Medical and approved by FDA in 2014 comprises a stimulation cuff electrode (standard bipolar stimulator), a pleural pressure sensing lead and an implantable pulse generator. Information from the pleural lead are sent to activate the stimulation cuff during the expiratory phase and stimulation is given between end-expiration and inspiratory period. The surgical procedure comprises three steps. The first step begins with a submandibular incision in order to identify submandibular gland and digastric tendon that are lift and retracted in order to visualize the milohyoid muscle that retracted, allows the vision of the hypoglossal branches (medial and lateral). The stimulation cuff is located in contact with the medial branches previously recognized by a nerve integrity monitoring system. The second step is the placing of the pleural sensor through a horizontal incision at the right fourth or fifth intercostal space lateral to the nipple line. The sensor is introduced in a pocket that is tunneled postero-anteriorly between the external and internal intercostal muscle layers since it brought into pleural contact. The third surgical step is creating a pocket for pulse generator 2 to $5 \mathrm{~cm}$ inferior to the right clavicle and medial to the delto-pectoral groove, deep to the subcutaneous fat and superficial to the pectoralis major muscle fascia. Inferiorly and superiorly, the pocket is extended by tunneling subcutaneously to the pleural sensing electrode and to the hypoglossal nerve stimulation cuff. Once the surgery is concluded the system is set to obtain an adequate tongue protrusion phasic with the breathing cycle.

As reported by Strollo et al severe complications rate is less that $2 \%$. Other proposed instrument give the possibility of reduce the number of implanted parts to the stimulation electrode like in LivaNova. ${ }^{38}$ The posttreatment presence of tongue stiffness, abrasions and postoperative swelling can be reduced adjusting stimulation parameters and by using mouth guards. ${ }^{38}$

\section{MAXILLO-FACIAL SURGERY}

Introduced in the latest 1980 for severe OSA patients, bimaxillary advancement consist in a Le Fort I osteotomy of the maxilla and bilateral sagittal split osteotomy of the mandible. After osteotomy the maxillomandibular massif is rotated in a counterclockwise direction and a reverse $\mathrm{T}$ mandibular osteotomy, consisting in a genioglossal advancement and advancing genioplasty, is made. Screws and plaque stabilize the segments. Piriform rim or anterior spine reduction surgery can complete the procedure. Changing in facial profile and maintenance of a functional occlusal stability influence the advancement that at least reaches $10-12 \mathrm{~mm} .{ }^{39}$ Whereas the procedure acts on bony structures the results are more stable in time despite the fact that the longest follow up reported in literature is 43.7 months. ${ }^{40}$

\section{DISCUSSION}

Therapeutic conscious orientation is objectively difficult in OSAS surgical scenario if we consider the great amount of surgical options and the continuous developing techniques. The result of the increasing interest and knowledge of anatomical basis and pathophysiology is the presence of several surgical solutions. The risk of the ever-growing amount of procedures can bring much variability when choosing the appropriate therapy. Above all is important to emphasize that most recent alternative is not always the best choice for all the patients. To avoid hazard of an ineffective treatment, specialists have refined the diagnostic approach and upraise the interpretation of anatomical findings. Since its introduction in clinical practice drug induced sleep endoscopy has been recognized as an helpful procedure that allow the physician to identify the collapsible site miming natural sleep in the operating room. Drug induced sleep endoscopy procedure, even via the study of treatments failure, have introduced the concept of multilevel collapse. ${ }^{8}$ A process of rethinking involves also nasal surgery that lead to reduce its therapeutic value in OSAS treatment but consider it as a step of a multilevel surgery or in order to improve ventilation compliance. $^{11}$ 
Probably the velo-uvulo-pharyngeal surgery is the head and neck surgical field in which changes perspectives have occurred more rapidly and more numerous. In Fujita's description of the UPPP, for instance, the preoperative oropharyngeal description regard only the presence of 'redundant tissue' with no further detailed description of the closure pattern. ${ }^{16}$ The description of 'Roman blinds or VULP' the technique introduce the decomposition into vectors of closure patterns and the anchorage of the soft tissue to bony structures. In Mantovani's description there are still terms like 'enlarge' and 'advance' with no objective quantification, however the embryonal new process of decomposing in vectors, rapidly evolves and lead to more tailored type of correction. Another important concept that lays the foundation of a new course in surgery is the anchorage of pharyngeal soft tissue to bony structures giving the possibility of an almost complete and durable correction in comparison to previous procedures. ${ }^{20-22}$ Though that basic changes, over the years, the aim has been to reduce the surgical aggressiveness to achieve the best results while minimizing the amount of damage.

Furthermore enhancing the efficacy of technologies and introducing tools conveyed from other specialties promoted procedural innovation and new perspectives.

One of the most widely used tool is the barbed wire. ${ }^{24}$ Its introduction has allowed more precise surgery, in particular made possible the correction of a specific collapse vector, giving the opportunity of a minimally invasive surgery. Preserving the correct physiological function of the oropharyngeal segment is, of course, the most important goal to achieve in every procedure.

Over the past decade, the global spread of the DaVinci system has revolutionized the approach fo the hypopharyngeal surgery. Burdened by a long preparatory phase (docking), the surgeon is allowed to clearly expose the target area and, even in this case, working in a more accurate and short manner. Procedures realized on epiglottis or tongue base are widely changed enabling access to the surgical site without need for conventional cervical incision. Previous procedures, like tongue base reduction with hyoepiglottoplasty, moreover were at probable risk of surgical lesion because of the cervical approach. The advantage of the trans oral approach reduces surgical duration, morbidity and damage to surrounding tissue. Furthermore it ensure a more rapid healing of patients with lower incidence of complications. ${ }^{28}$

Micro-technology and miniaturization are undergoing rapid developments that probably, over the next years, will increase the number of patient treated with non surgical therapies. The possibility of a tailored surgery and long lasting results raised the surgical option to a high level reducing the gap with medical therapies. Hypoglossal stimulation, for instance, is a method that changed considerably since it was introduced in clinical practice. ${ }^{38}$ The evolution of this system will lead to the creation of a component that can maintain the performances of the previous models with a reduced surgical approach.

Conversely some procedures show a relative stability. One of those is maxillo-mandibular advancement, in which only minimal concrete changes where introduced. But instead a shift in aesthetical sensitivity was registered being the intervention one with the strongest impact considering the external morphological changes. ${ }^{39}$

\section{CONCLUSION}

OSA syndrome is a complex pathology that request multidisciplinary evaluation. The surgical approach to the OSAS patient should be correctly and deeply studied with objective examination and DISE in order to tailor a therapy that improves symptoms and minimize the complications. In the past years countless surgical options have been encoded. Orientate oneself in this hard field would be difficult: for this wide knowledge of indication and purpose of the most frequently proposed procedure is mandatory. Technologic improving and new surgical procedures will appear in the future and would change and expand sleep surgery scenario.

\section{Funding: No funding sources \\ Conflict of interest: None declared \\ Ethical approval: Not required}

\section{REFERENCES}

1. Epstein LJ, Kristo D, Strollo PJ, Friedman N, Malhotra A, Patil SP, et al. Clinical guideline for the evaluation, management and long-term care of obstructive sleep apnea. J Clin Sleep Med. 2009;5:263-76.

2. Kapur VK, Auckley DH, Chowdhuri S, Kuhlmann DC, Mehra R, Ramar K, et al. Clinical practice guideline for diagnostic testing for adult obstructive sleep apnea: an American Academy of Sleep Medicine clinical practice guideline. J Clin Sleep Med. 2017;13(3):479-504.

3. Bonsignore MR, Baiamonte P, Mazzuca E, Castrogiovanni A, Marrone O. Obstructive sleep apnea and comorbidities: a dangerous liaison. Multidiscipl Respir Med. 2019;14:8.

4. Sullivan CE, Issa FG, Berthon-Jones M, Eves L. Reversal of obstructive sleep apnoea by continuous positive airway pressure applied through the nares. Lancet. 1981;1(8225):862-5.

5. Sutherland K, Kairaitis K, Yee BJ, Cistulli PA. From CPAP to tailored therapy for obstructive sleep Apnoea, Sutherland et al. Multidiscipl Respir Med. 2018;13:44.

6. Hall M. The instrument for tracheotomy. Lancet. 1848;2:530.

7. Kuhl W, Doll E, Franck MC. Successful management of Pickwickian syndrome using long- 
term tracheostomy. Dtsch Med Wochenschr. 1969;94(24):1286-90.

8. Croft CB, Pringle M. Sleep nasendoscopy: a technique of assessment in snoring and obstructive sleep apnoea. Clin Otolaryngol Allied Sci. 1991;16(5):504-9.

9. Meyer W. On adenoid vegetations in the nasopharyngeal cavity, their pathology, diagnosis and treatment. Hospitalstidende Copenhagen. $1868 ; 11: 177-8$.

10. Betz F. On the snoring of very young children. Memorabilia Heilbronn. 1858;3:73-7.

11. De Vito A, Berrettini S, Carabelli A, SellariFranceschini S, Bonanni E, Gori S, et al. The importance of nasal resistance in obstructive sleep apnea syndrome: a study with positional rhinomanometry. Sleep Breath. 2001;5(1):3-11.

12. Cai Y, Goldberg AN, Chang JL. The nose and nasal breathing in sleep apnea. Otolaryngol Clin North Am. 2020;53(3):385-95.

13. Vicini C, De Vito A, Benazzo M, Frassineti S, Campanini A, Frasconi $\mathrm{P}$, et al. The nose oropharynx hypopharynx and larynx (NOHL) classification: a new system of diagnostic standardized examination for OSAHS patients. Eur Arch Otorhinolaryngol. 2012;269(4):1297-300.

14. Ikematsu T. Study of snoring: therapy. J Japan Otol Rhinol Laryngol Soc. 1964;64:434-5.

15. Quesada P, Botet J, Fuentes E, Perelló E. Partial resection of the soft palate as treatment for hypersomnia and breathing syndrome in obese patients. ORL Dips. 1977;5:81-8.

16. Fujita S, Conway W, Zorick F, Roth T. Surgical correction of anatomic abnormalities in obstructive sleep apnea syndrome: uvulopalatopharyngoplasty. Otolaryngol Head Neck Surg. 1981;89(6):923-34.

17. Ellis PD. Laser palatoplasty for snoring due to palatal flutter: a further report. Clin Otolaryngol Allied Sci. 1994;19(4):350-1.

18. Pang KP, Terris DJ. Modified cautery-assisted palatal stiffening operation: new method for treating snoring and mild obstructive sleep apnea. Otolaryngol Head Neck Surg. 2007;136(5):823-6.

19. Cahali MB. Lateral pharyngoplasty: a new treatment for obstructive sleep apnea hypopnea syndrome. Laryngoscope. 2003;113(11):1961-8.

20. Pang KP, Woodson BT. Expansion sphincter pharyngoplasty: a new technique for the treatment of obstructive sleep apnea. Otolaryngol Head Neck Surg. 2007;137(1):110-4.

21. Pang KP, Piccin O, Pang EB, Pang KA, Chan YH, Rotenberg BW. Combined expansion pharyngoplasty and anterior palatoplasty for the treatment of OSA. Indian J Otolaryngol Head Neck Surg. 2016;68(4):528-533.

22. Mantovani M, Minetti A, Torretta S, Pincherle A, Tassone G, Pignataro L. The velo-uvulo-pharyngeal lift or "roman blinds" technique for treatment of snoring: a preliminary report. Acta Otorhinolaryngologica Italica. 2012;32:48-53.
23. Salamanca F, Costantini F, Mantovani M, Bianchi A, Amaina T, Colombo E, et al. Barbed anterior pharyngoplasty: an evolution of anterior palatoplasty. Acta Otorhinolaryngologica Italica. 2014;34:434-8.

24. Vicini C, Hendawy E, Campanini A, Eesa M, Bahgat A, AlGhamdi S, et al. Barbed reposition pharyngoplasty (BRP) for OSAHS: a feasibility, safety, efficacy and teachability pilot study. "We are on the giant's shoulders". Eur Arch Otorhinolaryngol. 2015;272(10):3065-70.

25. Mantovani M, Carioli D, Torretta S, Rinaldi V, Ibba T, Pignataro L. Barbed snore surgery for concentric at the velum collapse: the Alianza technique. J Cranio-Maxillofac Surg. 2017;45(11):1794-800.

26. Djupesland G, Schrader H, Lyberg T, Refsum H, Lilleås $\quad \mathrm{F}, \quad$ Godtlibsen Palatopharyngoglossoplasty in the treatment of patients with obstructive sleep apnea syndrome. Acta Otolaryngol Suppl. 1992;492(492):50-4.

27. Kaya N. Sectioning the hyoid bone as a therapeutic approach for obstructive sleep apnea. Sleep. 1984;7(1):77-8.

28. Chabolle F, Wagner I, Blumen MB, Séquert C, Fleury B, De Dieuleveult T. Tongue base reduction with hyoepiglottoplasty: a treatment for severe obstructive sleep apnea. Laryngoscope. 1999;109(8):1273-80.

29. Powell NB, Riley RW, Guilleminault C. Radiofrequency tongue base reduction in sleepdisordered breathing: A pilot study. Otolaryngol Head Neck Surg. 1999;120(5):656-64.

30. Vicini C, Dallan I, Canzi P, Frassineti S, La Pietra MG, Montevecchi F. Transoral robotic tongue base resection in obstructive sleep apnoea-hypopnoea syndrome: a preliminary report. ORL 2010;72:22-7.

31. Vicini C, Montevecchi F, Pang K, Bahgat A, Dallan I, Frassineti S, et al. Combined transoral robotic tongue base surgery and palate surgery in obstructive sleep apnea-hypopnea syndrome: Expansion sphincter pharyngoplasty versus uvulopalatopharyngoplasty. Head Neck. 2013;36:77-83.

32. Andersen APD, Alving J, Lildholdt T, Wulff $\mathrm{CH}$. Obstructive sleep apnea initiated by a lax epiglottis. A contraindication for continuous positive airway pressure. Chest. 1987;91(4):621-3.

33. Fearon B, Ellis D. The management of long term airway problems in infants and children. Ann Otol Rhinol Laryngol. 1971;80(5):669-77.

34. Lane RW, Weider DJ, Steinem C, Marin-Padilla M. Laryngomalacia. A review and case report of surgical treatment with resolution of pectus excavatum. Arch Otolaryngol. 1984;110(8):546-51.

35. Salamanca F, Leone F, Bianchi A, Bellotto R, Costantini F, Salvatori P. Surgical treatment of epiglottis collapse in obstructive sleep apnoea syndrome: epiglottis stiffening operation, Acta Otorhinolaryngologica Italica. 2019;39:404-8. 
36. Browaldh N, Markstrom A, Friberg D. Elective tracheostomy is an alternative treatment in patients with severe obstructive sleep apnoea syndrome and CPAP failure. Acta Oto-Laryngologica. 2009;129:1121-6.

37. Costantino A, Rinaldi V, Moffa A, Luccarelli V, Bressi F, Cassano M, et al. Hypoglossal nerve stimulation long-term clinical outcomes: a systematic review and meta-analysis. Sleep Breath. 2019:1-3.

38. Strollo PJ, Soose RJ, Maurer JT, Upper-airway stimulation for obstructive sleep apnea. N Engl J Med. 2014;370:139-49.

39. Ohen-Levy J, Petelle B, Vieille E, Dumitrache M, Fleury B. Changes in facial profile after maxillomandibular advancement surgery for obstructive sleep apnea syndrome. Int Orthodont. 2013;11:71-92.

40. Zaghi S, Holty JE, Certal V, Abdullatif J, Guilleminault C, Powell NB, et al. Maxillomandibular advancement for treatment of obstructive sleep apnea: a meta-analysis. JAMA Otolaryngol Head Neck Surg. 2016;142(1):58-66.

Cite this article as: Sparacino L, Bruschini L, De Vito A, Fiacchini G, Berrettini S. Surgical treatment in obstructive sleep apnea syndrome: an overview. Int Surg J 2020;7:2807-13. 